\title{
Embedding biobanks as tools for personalised medicine
}

\author{
Jane Kaye \\ Centre for Health, Law and Emerging Technologies (HeLEX), Department of Public Health, \\ University of Oxford, Old Road Campus, Headington OX3 7LF, UK \\ E-mail: jane.kaye@law.ox.ac.uk
}

\begin{abstract}
Many governments are seeking to improve the translation of medical research innovation by developing mechanisms to aid the flow of information between the laboratory and the clinic. The intention is that the combination of new research insights and the use of next generation sequencing technology in the clinic will lead to more and better personalised medicine outcomes. However, existing healthcare structures often maintain the sharp line between research and the clinic, which slows down the bidirectional flow of information that is needed to enable effective translation. At the same time, within the biobanking field, infrastructures are being built to enable more efficient and better quality research. As with translational research, there are a number of challenges for these biobanking initiatives. Questions remain as to the best way to utilise biobanks for translational research. Other challenges are how to ensure the sustainability of individual biobanks and the biobank infrastructures that are being developed. Sustainability requires public support for long-term funding, as well as the on-going commitment of patients and research participants to provide the raw material for biobanks and regular use by researchers. One possible solution to the challenges that are found in these two areas is to embed a biobank within the healthcare structure and to use it for healthcare and research purposes simultaneously. This paper discusses the CuraRata and the EnCoRe dynamic consent models that in combination could provide sustainability for biobanks and at the same time enable translational research.
\end{abstract}

The National Human Genome Research Institute (NHGRI) vision for genomics published in Nature [1] in February 2011, highlighted the importance of biobanks to support translational research and the development of genomic medicine. While acknowledging that advances in genomic medicine (clinical care based on genomic information) have been few [2-6], the paper anticipates that from 2020 this could radically change with new innovations having greater clinical application and personalised medicine becoming more routine. Reaching this goal will depend upon the further development of existing infrastructure, such as biobanks, as well as the assembly of data sets of sequence information of higher quality and comprehensiveness than currently exists and the linkage of this information to data gathered in the course of actual clinical care, such as in electronic medical/health records [7]. However, as the paper acknowledges, moving from a vision statement into implementation, is notoriously difficult and will involve considerable effort and time by many people in different disciplines. The purpose of this paper is to present a translational research model that embeds a biobank into the healthcare structure as a first step to enable greater translation in personalised, genomic medicine. This model is based on the CuraRata model being developed in Leiden in the Netherlands and is one that is being assessed for use at the University of Oxford in the UK. The aim of this model is to enhance the possibilities for innovation in translational research and personalised medicine. This paper will firstly describe some of the current challenges in translational medicine and biobanking that may be solved by using such a model and then articulate how embedding biobanks within clinical care may lead to better translational research opportunities.

\section{CHALLENGES IN TRANSLATIONAL MEDICINE}

There has been considerable investment in improving translational medical research outcomes, and leading examples can be found in the USA, Canada, the Netherlands, Singapore and the UK. There are many definitions of 'translational research' but at its simplest it means taking the findings from laboratory research and implementing these in a clinical setting - from 'bench to bedside'. The investments in translational research initiatives have been spurred by the realisation that 'the creation of novel treatments remains protracted and expensive' [8], new discoveries are not delivered swiftly to patients [9-11], and population-wide strategies using cheap, simple, and efficient interventions are not effectively implemented [12]. However, this is not simply a question of creating new knowledge. New discoveries have not automatically led to improvements in healthcare. Although we have seen considerable advances in understanding the molecular, genetic and cellular origins of disease, there has not been the capacity within the current scientific and clinical structures to turn these into "new paradigms to improve the health and quality of life of individuals, communities and populations' [13].

One of the reasons advanced for the poor track record in translational research is that the current interface between the research and clinical domains does not allow for the bidirectional flow of knowledge between the bench and the bedside that could lead to innovation [14]. Within most jurisdictions there are different regulatory regimes for healthcare and for research, and these do not necessarily lead to an integrated approach for translating knowledge into healthcare benefit for patients. Research and treatment 
are largely carried out under different institutional frameworks and are based on different professional cultures, regulations and guidelines. There are also significant differences between the practices of public institutions and commercial entities. Translational research often fits in the grey area between these two regimes. As a result, there can be a clash of cultures, or a lack of multidisciplinary understanding which can make the verification of findings and the translation of research results difficult [15]. Government policies are aimed at trying to make the relationship between healthcare and research closer, and the translation of research results faster and more efficient. However, these different regulatory domains mean that innovations can still take considerable time before they are clinically implemented.

To enhance the possibilities for translational research, it has been argued that there needs to be a more intimate interface between the research and clinical domains [16]; a transformation in professional culture that rewards science practiced as part of interdisciplinary teams, rather than the current silos that reward individual contributions [17]; and the investment in informatics to enable disparate biobanks and data systems to be used for research as well as to inform operational and clinical decision making [18]. Advances in IT could facilitate this, as informatics provides the possibility to link, mine and use datasets for both clinical and research purposes simultaneously which has the potential to enhance translational research opportunities. For translational research to become more routine there also needs to be a paradigm shift from a focus on the development of 'new therapeutic modalities for disease palliation, to the assessment of risk and the science of wellness and disease prevention' [19] and a move towards the realm of personalised healthcare solutions. This requires a reformulation of the relationships between health care providers and research institutes, as well as between patients, research participants and researchers; greater harmony between different regulatory regimes; and a cultural change to align clinical and research activities more closely with translational objectives.

\section{Challenges IN BIOBANKING}

Over the past ten years, considerable effort has been put into the development of tools - software, protocols, procedures and governance mechanisms - required to establish and run biobanks as resources for research by a number of organisations, such as $\mathrm{P}^{3} \mathrm{G}$ (www.p3g.org), biobanking societies such as ISBER (www.isber.org), ESBB (www.esbb.org/), The Marble Arch Working Groups (www.oncoreuk.org), and IARC (www.iarc.fr). European-funded projects such as PHOEBE and BBMRI (http://www.bbmri.eu/) have focused specifically on developing harmonisation and standardisation tools for biobanks. These activities in combination have had a considerable effect on the development of best practice in the biobanking field.

Now, as a number of biobanks have been established de novo, we are moving into a new phase of infrastructure development where existing disease-based research collections are being networked through a 'hub and spokes' model in member states across Europe. The pan-Europe biobanking and biomolecular research infrastructure (BBMRI) aims to 'improve the accessibility and interoperability of the existing comprehensive collections, either population-based or diseaseorientated, of biological samples' and their associated information to build 'a globally unmatched, Europewide platform for translational medical research' [20]. Over the past years, these plans have gained momentum as 9 countries across Europe have made a total investment of $€ 97.8 \mathrm{~m}$ to fund national BBMRI infrastructures to co-ordinate biobanking at a national level. Some of these biobanks have been developed through the clinic and others are the result of research activities. However, one of the challenges for all of these initiatives is how to recover the costs of curating and maintaining the BBMRI coordinating structures and individual biobanks in the long term.

Another challenge as we move to these 'networks within networks' of biobanks is how to engage the public and to protect the interests of participants who have contributed to the various clinical collections, research projects and biobanks that will be a part of these wider networks. Our current governance systems for research are nationally based with oversight primarily by institutional research boards (IRB's) or research ethics committees (RECs) in conjunction with other government bodies. In all jurisdictions there are legal requirements embedded in human rights or information law, as to how to protect the privacy of patient and research participants. One of the basic principles for research is that consent from the individual must be obtained before the research commences and this is also a condition of privacy and information law. There also exist a number of exemptions to this basic principle for different types of medical research. In the case of biobanks, the only practical solution has been to obtain a broad consent to future research uses, as all research uses and researchers cannot be obtained at the time of recruitment. This trend is still highly debated in the bioethics community [21]. Best practice in biobanking requires that information and samples are also coded to protect the identity and privacy of individuals, particularly if information is shared. There are a number of examples of biobanks that are used for translational research which rely on anonymisation and coding techniques and opt out consent (e.g. UCLA CTSI: http://www.ctsi.ucla.edu/, Washington University Institute for Clinical and Translational Sciences: http://icts.wustl.edu/). Both coding and anonymisation are often used as a way to protect individuals when consent for new research uses maybe difficult to obtain. As wide-scale data sharing increases through the building of biobanking infrastructure and the use of 
next generation sequencing technologies become more wide spread, it is increasingly difficult to anonymise data and this raises concerns about how best to protect privacy [22]. Privacy is often associated with control over the use of personal information as described by Lord Justice Law of the English Court of Appeal:

... subject to [certain] qualifications ... an individual's personal autonomy makes him - should make him - master of all those facts about his own identity, such as his name, health, sexuality, ethnicity, his own image ... and also of the 'zone of interaction' ... between himself and others. He is the presumed owner of these aspects of his own self; his control of them can only be loosened, abrogated, if the State shows an objective justification for doing so (Wood v Commissioner of Police for the Metropolis [2009] EWCA Civ 414 at §21).

Medical research is often regarded as one area where exemptions to this human right can be justified. However, there is increasing empirical research showing that individuals want to know how their personal information is being used and they want to be asked for consent in the case of data sharing [23]. How much control an individual may want over their personal information can be context specific and will depend upon the degree of public trust that an institution or biobank has. Therefore, careful attention needs to be given to the on-going relationship between research participants and a biobank.

Establishing and maintaining a biobank requires long-term commitment and wide-scale support from the public in order to sustain the high participant levels that are required, ensure public trust and to recruit participants who are willing to be re-contacted for involvement in future research projects. This has also been a significant challenge for clinical trials used for personalised medicine that must screen a much larger pool of potential participants to identify those that have the characteristics of the disease and this means that patient recruitment has been a major stumbling block in getting trials completed on time [24,25]. In the case of translational research, governance mechanisms need to be developed to facilitate recruitment for research within a clinical setting; to establish management pathways to feed back research results and incidental findings to individuals, [26-28] to recruit people into additional studies; to develop mechanisms to establish partnerships in research; establish what patients want to know about the research conducted on their samples and information; and whether they are concerned about ownership issues [29-31] and commercialisation [32,33]. A more dynamic relationship is needed between participants and the research community to address these concerns, and new models of governance based on participant involvement need to be developed [34-36].

The sustainability of biobanks will also depend upon having a resource that is attractive to researchers. This means that the biobank must hold high quality, well-characterised samples and accompanying data that can be used for many different research studies. The attractiveness of a resource for research purposes also requires access approval systems that are not unduly bureaucratic but have a quick turn around and can fast track low-risk research proposals. In addition, appropriate rewards and incentives need to be developed for clinicians and researchers to collect samples and information to continue to maintain the resource. There are a number of examples of best practice in biobanking, but two current and related issues still need to be resolved. These are the development of access policies with cost recovery pricing models and how to recover the costs of running a biobank once the initial funding has ceased.

Developing a sustainable business model for biobanks has to incorporate some incentives for collectors by covering the costs of curation but also offer rewards for innovation. Attention needs to be given to developing intellectual property models and allowing access to pre-competitive data while still enabling innovation to be rewarded. Wide scale data sharing rationales can conflict with intellectual property frameworks, which are traditionally regarded as important for translation. Views on the appropriate role of intellectual property in innovation can be polarised, with some believing that intellectual property rights are necessary for translation and others believing that intellectual property creates monopolies, limits competition, and allows patentees to establish high prices, resulting in global inequalities in terms of access to drugs [37]. For new research infrastructure or biobanks, embedded in a research context where open access and data sharing are promoted as being drivers of innovation, there is a need to move away from the business model of establishing high intellectual property barriers. Intellectual property rights can be used as tools to sustain and maintain innovation through building new models of collaboration and trust [38]. However, there has been little research to understand how this might be done in the field of genomics and biobanks.

\section{THE MODEL}

The CuraRata model [39] in the Netherlands is designed to promote translational research by embedding a biobank within the healthcare structure. In this model, information and samples derived from a clinical setting can also be used for research purposes, but in turn be used to guide prevention, diagnosis and treatment. As well as enabling translational research, this model also provides a solution to some of the challenges that have been identified in the biobanking field, such as financial sustainability and ensuring the viability and attractiveness of the biobank for researchers and industry. In combination with the EnCoRe patient interface it also provides the basis to address privacy concerns by placing patients at the core of the translational pathway. The discussion that follows considers how the CuraRata model in combination with EnCoRe 'dyna- 


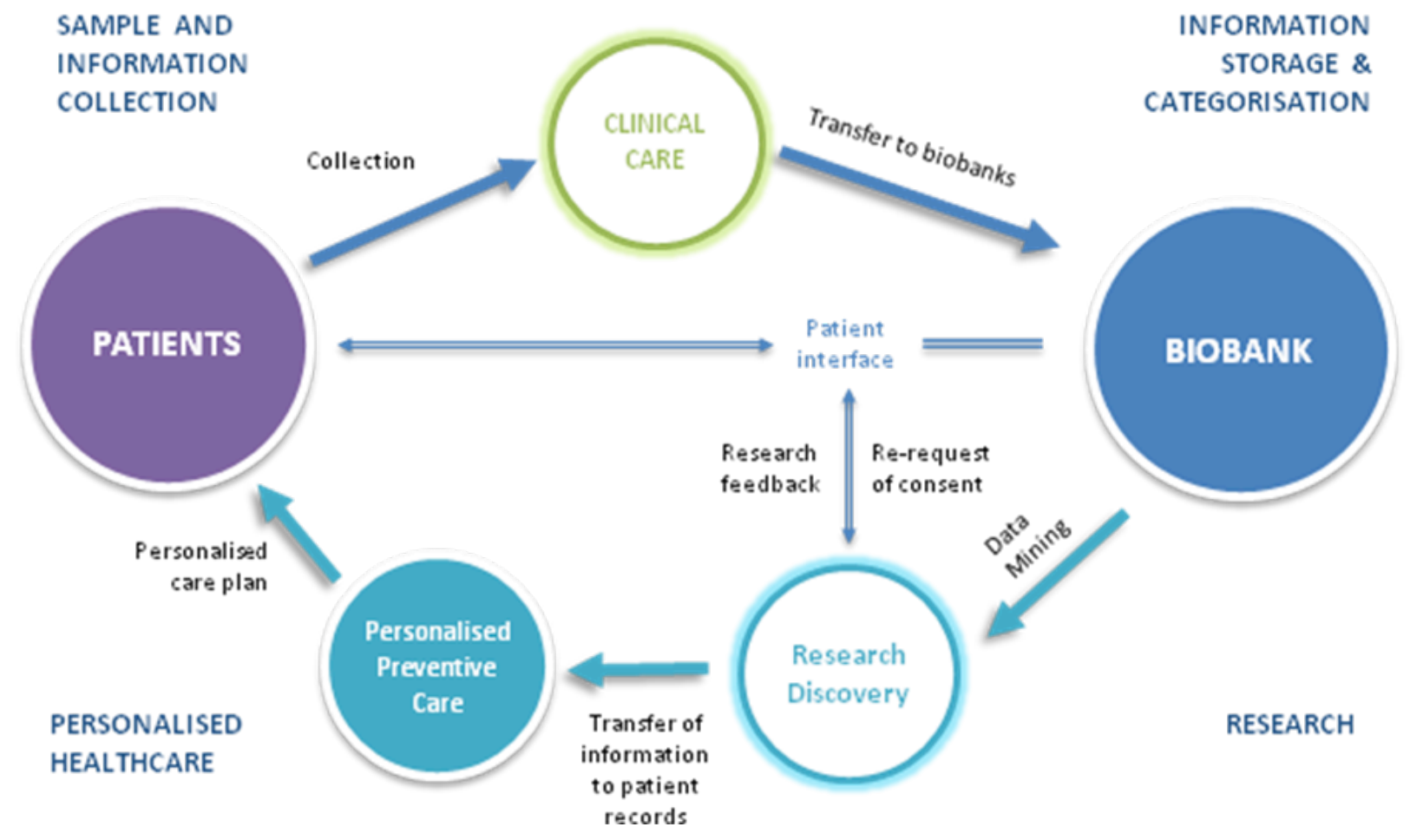

Figure 1.

mic consent' patient interface, might be a suitable model to embed a biobank within a university research hospital setting (Fig. 1).

This model firmly anchors the biobank within the healthcare structure with the dual purpose of being a tool for translational research but also for clinical care and potentially personalised medicine. Patients are central to this model, as they are the suppliers of samples and data for research purposes but they are also the focus of healthcare delivery. Personal medical data and samples gathered routinely as part of the clinical diagnosis and treatment process can be accumulated in a linked electronic record system and biobank to be accessed for research purposes in real time. This bidirectional flow of data and samples has the potential to bring the interface between the clinic and research closer together so that the possibilities for translational research are increased. Figure 1 (adapted from the CuraRata literature) illustrates how information flows from the clinic through the biobank and then can be used in the clinic for personalised medicine approaches.

The EnCoRe 'dynamic consent' interface [40] enables research participants to have a dynamic, interactive relationship with the custodians of biobanks and the research community - what we call a 'dynamic consent' - using web 2.0 technologies. This is in contrast to the one off broad consent that is currently the only practical solution for many projects or biobanks. Rather than researchers having to anonymise research data to enable secondary research that was not envisaged at the time of collection, using EnCoRe, the custodian of the biobank can directly contact a donor for informed consent for this unanticipated new research use. This ensures that the highest ethical and legal standards are maintained, which in turn supports high levels of research integrity, patient confidence and public trust. This software can facilitate recruitment for research within a clinical setting; provide management pathways to feed back research results and incidental findings into the clinical record; recruit people into additional studies; and provide patients with information about the research conducted on their samples and information. The use of dynamic consent interface allows patient samples and data to be tracked across research studies to remove bias and erroneous identification, provides a mechanism for re-contacting individuals for recruitment into new studies, and could be used as a basis for cutting down on research ethics oversight for secondary research.

\section{IMPLEMENTATION CHALLENGES}

To implement the CuraRata and EnCoRe models will require a clear strategy, resources and significant changes to existing practice, procedures and governance structures. This is because most hospital and clinical settings are designed to keep treatment and research as distinct areas, even though this is detrimental to translation. These requirements are enshrined in law and enforced by a number of governance procedures, practices and policies. The possibility that all people entering the hospital or clinic become part of a cohort, and 
are given a baseline assessment that can be used for clinical care but also for research purposes, changes the relationship between the patient and health care professionals. To ensure that this is clearly understood by patients will require a series of public engagement activities so that people are aware of how their information will be used but also to ensure that there is strong community support for such a change in practice and orientation. Establishing what patients want to know about the research conducted on their samples and information; and whether they are concerned about ownership issues [29], and commercialisation [32] will be essential. Such engagement will help to foster the long-term commitment and wide-scale support from the public in order to maintain high participant levels, engender their trust and to ensure that there are participants who are willing to be re-contacted for involvement in future research projects.

Patients will need to know that personal information will be accessible to third party researchers that will include commercial partners and that because of the richness of the data and samples held in the biobank they may be 'distinguished', but not necessarily identified, in the data set from other patients [41]. However, because of the dynamic consent model, patients will be able to be informed about all the uses of their data, so that they will know how their data are being used and who is using it. This level of transparency and accountability, as well as assurances that access will only be permitted by bonafide professionals bound by contractual agreements of confidentiality, will encourage trust and participation. Therefore, for patients the issue of being distinguished in a data set rather than being anonymous, may not be of great concern because of the safeguards in place for data security, and that the use of the dataset will have benefits for others as well as their own healthcare. Explaining how data will be used and by whom is also in conformity with best principles of data protection legislation and privacy law. However, the exact details of how data is used and under what conditions, will need to be determined for each institution as ideas about acceptable levels of privacy breaches are context specific. Therefore, implementation will require careful consultation and engagement with patients as well as healthcare professionals to develop models of best practice.

To use the dynamic consent model and embed a biobank in the healthcare structure, will require that flows of patient information are redesigned and protocols are developed to ensure that samples and patient information can be used for both clinical and research uses while at the same time the interests of patients are protected. Policies and protocols are needed to determine who can have access to such data and for what purposes, and to articulate the transparent and accountable oversight structures to protect patient privacy and ensure fair access to the biobank samples and information. This requires the inclusion of patients in key decision-making bodies to enable flexible decision making that can address the wider concerns of the community. Care will also need to be taken to develop partnerships with industry so that innovation can be encouraged as well as the commercialisation of findings and results.

One of the biggest challenges will be aligning the governance mechanisms to enable the bidirectional flow of information and samples through the biobank into either the clinical or research domains. The current ethical and legal differences between these domains make it difficult for translational research strategies. The effect of these differences must be carefully understood and articulated before any changes are made to bring them into alignment. It is important that these different areas maintain their contextual integrity, but that they can be pulled together, like the teeth in a zipper, as integral parts of this translational research model. To enable this, governance mechanisms need to be developed to facilitate recruitment to the biobank and research within a clinical setting; to establish management pathways to feed back research results and incidental findings to individuals [28], to recruit people into additional studies once they are a member of the biobank; to develop mechanisms to establish partnerships in research between clinicians and researchers; professionals, patients and the community. This also requires an institutional cultural change to align both the clinic and research activities more closely with translational objectives. These complex organisational, operational and governance challenges are not yet fully articulated or understood and require further investigation and attention.

\section{CONCLUSION}

Embedding a biobank within a healthcare setting has the potential to address many of the challenges that have been identified in the translational research setting and in the biobanking sector. Such a model enables the bidirectional flow of information between the clinic and the research context to enhance translational research. It also addresses some of the issues of on-going recruitment and sustainability of the biobank. However, to achieve this vision requires new kinds of partnerships, governance, organisational and administrative structures to align the clinical and research activity towards translational research objectives. This is not an easy task and will require institutional commitment, cultural and organisational change and the formulation of new requirements for the grey area of translational research and IT resources to enable this to be implemented. Further pilot studies are needed to test this model so that we can start to fully capitalise on our existing resources and increase the possibilities for advances in knowledge and the way that healthcare is delivered. 


\section{ACKNOWLEDGEMENTS}

The idea of embedding a biobank within clinical care was first suggested in the biobanking context at the final panel of the Harmonizing Biobank Research - An Achievable WorldWide Goal conference co-organised by PHOEBE (Promoting Harmonization of Epidemiological Biobanks in Europe), P3G (Public Population Project in Genomics) and BBMRI (Biobanking and Biomolecular Resources Research Infrastructure) held on the 25th - 27th March 2009 in Brussels,
Belgium. I would like to thank Daniel Hommes of the University Medical Center, Leiden, The Netherlands for generously sending me his slides that mentioned the CURATATA project, and my colleagues Rachel Magher, James Shepherd, Runjan Chetty, Karen Melham (University of Oxford). I would also like to thank Colin Mitchell for compiling Fig. 1 and helping to format this ready for publication. This research has been funded by the Wellcome Trust grant number WT081407/Z/06/Z and the TSB/ESRC/EPSRC EnCoRe Project EP/G002541/1.

\section{REFERENCES}

1. Green ED, Guyer MS, National Genome Research Institute. Charting a course for genomic medicine from base pairs to bedside. Nature 2011; 470: 204-13.

2. Paik S, et al. A multigene assay to predict recurrence of tamoxifen-treated, node-negative breast cancer. $N$ Engl J Med 2004; 351: 2817-26.

3. Tozzi V. Pharmacogenetics of antiretrovirals. Antiviral Res 2010; 85: 190-200.

4. Angiolillo DJ, et al. Contribution of gene sequence variations of the hepatic cytochrome P450 3A4 enzyme to variability in individual responsiveness to clopidogrel. Arterioscler Thromb Vasc Biol 2006; 26: 1895-1900.

5. Rieder MJ, et al. Effect of VKORC1 haplotypes on transcriptional regulation and warfarin dose. $N$ Engl $J$ Med 2005; 352: 2285-93.

6. Goetz MP, et al. The impact of cytochrome P450 2D6 metabolism in women receiving adjuvant tamoxifen. Breast Cancer Res Treat 2007; 101: 113-21.

7. Feero WG, Guttmacher AE, Collins FS. Genomic medicine - An updated primer. N Engl J Med 2010; 362: 2001-11.

8. Califf RM, Peterson ED, Gibbons RJ, et al. Integrating quality into the cycle of therapeutic development. $J$ Am Coll Cardiol 2002; 40: 1895-901.

9. Lee TH. Eulogy for a quality measure. $N$ Engl J Med 2007; 357: 1175-7.

10. Balas EA. Information systems can prevent errors and improve quality. J Am Med Inform Assoc 2001; 8: 398-9.

11. Bioscience Innovation and Growth Team: The Review and Refresh of Bioscience 2015. A Report to Government. UK Department for Business, Enterprise and Regulatory Reform, January 2009.

12. Califf RM, Peterson ED, Gibbons RJ, et al. Integrating quality into the cycle of therapeutic development. $J$ Am Coll Cardiol 2002; 40: 1895-901.

13. Feldman A. CTS: A new discipline to catalyze the transfer of information. Clin Transl Sci 2008: 1: 1-2.

14. Budin-Ljøsne I, Tassé AM, Knoppers BM, Harris JR. Bridging consent: from toll bridges to lift bridges? BMC Med Genomics 2011; 4: 69.

15. Talmon JL, Maurits GR, Legemate DA. PSI: The Dutch Academic Infrastructure for shared biobanks for translational research. Summit on Translat Bioinforma 2008: 110-4.

16. Schmittdiel JA, et al. System-based participatory research in health care: an approach for sustainable translational research and quality improvement. Ann Fam Med 2010; 8: 256-9.

17. Bonham AC, et al. Putting evidence to work: an expanded research agenda for academic medicine in the era of health care reform. Acad Med 2010; 85: 1551-3.

18. Harris PA, et al. Research electronic data capture (REDCap) - A metadata-driven methodology and workflow process for providing translational research informatics support. J Biomed Inform 2009; 42 (2): 377-81.

19. Clancy C, Collins FS. Patient-Centered Outcomes Research Institute: The intersection of science and health care. Sci Transl Med 2010; 2: $37 \mathrm{~cm} 18$.

20. BBMRI Brochure, October 2010.

21. Caulfield T, Kaye J. Broad consent in biobanking: reflections on seemingly insurmountable dilemmas. Med Law Int 2009; 10: 85-100.

22. Kaye J. Data sharing and privacy in genomic research. Annu Rev Genomics Hum Genet 2012; 13 (in press).

23. Trinidad SB, Fullerton SM, Ludman EJ, Jarvik GP, Larson EB, Burke W. Research ethics. Research practice and participant preferences: the growing gulf. Science 2011; 331 (6015): 287-8.

24. Ledford H. Translational research: 4 ways to fix the clinical trial. Nature 2011; 477: 526-8.

25. Maxmen A. Translational research: The American way. Nature 2011; 478: S16-8.

26. Forrest LE, Delatycki MB, Skene L, Aitken M. Communicating genetic information in families - a review of guidelines and position papers. Eur J Hum Genet 2007; 15: 612-8.

27. Marshall E. Human genetics in the clinic, one click away. Science 2011; 331: 528-9. 
28. Cho MK. Understanding incidental findings in the context of genetics and genomics. J Law Med Ethics 2008; 36: $280-5$.

29. Hardcastle R. Law and the Human Body: property rights, ownership and control. Oxford: Hart, 2007.

30. Harris PA, et al. Research electronic data capture (REDCap) - A metadata-driven methodology and workflow process for providing translational research informatics support. J Biomed Inform 2009; 42 (2): 377-81.

31. Mason JK, Laurie GT. Consent or property? Dealing with the body and its parts in the shadow of Bristol and Alder Hey. Mod Law Rev 2001; 64: 710-29.

32. Haddow G, Laurie G, Cunningham-Burley S, Hunter KG. Tackling community concerns about commercialisation and genetic research: a modest interdisciplinary proposal. Soc Sci Med 2007; 64: 272-82.

33. Hopkins MM, et al. The Patenting of Human DNA: Global Trends in Public and Private Sector Activity (The PATGEN Project). SPRU, Science and Technology Policy Research, University of Sussex, 2006.

34. Clayton E. Informed consent and genetic research. In: Rothstein M (ed). Genetic Secrets: Protecting Privacy and Confidentiality in the Genetic Era. New Haven, CT: Yale University Press, 1997: 126-36.

35. Mascalzoni D, Hicks A, Pramstaller P, Wjst M. Informed consent in the genomics era. PLoS Med 2008; 5: e192.

36. Green ED, Guyer MS, National Genome Research Institute. Charting a course for genomic medicine from base pairs to bedside. Nature 2011; 470: 204-13.

37. Zarocostas J. Huge inequalities in access to drugs partly because of intellectual property rights, says UN Expert. BMJ 2009; 338: b2348.

38. Piper T, Gold R. Practice, Policies and Possibilities in Licensing in Human Genetics. The Innovation Partnership for Health Canada, 2008.

39. http://www.curarata.nl/.

40. Kaye J. From single biobanks to international networks: developing e-governance. Hum Genet 2011; 130: 377-82.

41. Malin B, Karp D, Scheuermann RH. Technical and policy approaches to balancing patient privacy and data sharing in clinical and translational research. J Investig Med 2010; 58: 11-8. 